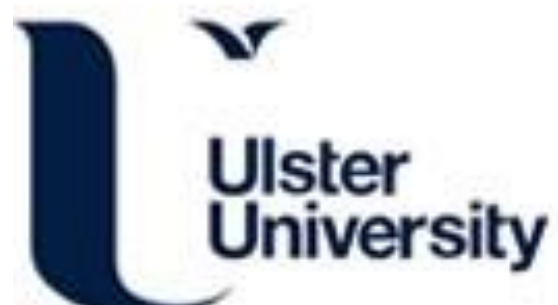

\section{Localised Transmission Power Adjustment for Relative Connectivity Awareness in Wireless Ad-hoc \& Sensor Networks}

Panousopoulou, A., Sterritt, R., \& Tzes, A. (2012). Localised Transmission Power Adjustment for Relative Connectivity Awareness in Wireless Ad-hoc \& Sensor Networks. In Unknown Host Publication (pp. 460-465). IEEE Control Society.

Link to publication record in Ulster University Research Portal

\section{Published in:}

Unknown Host Publication

Publication Status:

Published (in print/issue): 01/06/2012

\section{Document Version}

Publisher's PDF, also known as Version of record

\section{General rights}

Copyright for the publications made accessible via Ulster University's Research Portal is retained by the author(s) and / or other copyright owners and it is a condition of accessing these publications that users recognise and abide by the legal requirements associated with these rights.

\section{Take down policy}

The Research Portal is Ulster University's institutional repository that provides access to Ulster's research outputs. Every effort has been made to ensure that content in the Research Portal does not infringe any person's rights, or applicable UK laws. If you discover content in the Research Portal that you believe breaches copyright or violates any law, please contact pure-support@ulster.ac.uk. 


\title{
Localised Transmission Power Adjustment for Relative Connectivity Awareness in Wireless Ad-hoc \& Sensor Networks
}

\author{
Athanasia Panousopoulou, Roy Sterritt and Anthony Tzes
}

\begin{abstract}
Wireless Ad-hoc and Sensor Networks are the cornerstone of decentralised control and optimisation techniques in numerous sensor-rich application areas. Triggered by the necessity of autonomous operation within constantly changing environments, Wireless Ad-hoc and Sensor Networks are characterised by dynamic topologies, regardless the mobility attributes of their operational nodes. As such, the relative awareness that each node can obtain of the entire network draws the roadmap of viable reconfiguration mechanisms, such as the establishment of bidirectional connectivity. The issues addressed in this paper are related to the bidirectional connectivity conditions over Wireless Ad-hoc and Sensor Networks. Based solely on the relative awareness that each node has of the entire network, sufficient end-to-end connectivity conditions are herein extracted. These conditions, exploiting the notion of relative Delaunay neighbourhoods, formulate the basis of a transmission power adjustment scheme. Without any additional network overhead, the resulting Relative Delaunay Connectivity Algorithm is herein proven to yield an efficient solution to the connectivity issues. Extensive simulation results are offered to evaluate the performance of the network, resulting from the proposed transmission range adjustment, whilst highlighting the benefits of the Relative Delaunay Connectivity Algorithm.
\end{abstract}

\section{INTRODUCTION}

Wireless Ad-hoc and Sensor Networks (WASN) have gained prominence as a cross-disciplinary research field for numerous sensor-rich application areas. Driven by the needs of these applications, a WASN is expected to operate in a self-organised manner and adapt itself to variations of both the surrounding environment and the demands of the user [11]. Consequently, even in the case of static nodes, a WASN is characterised by dynamic topologies, where alterations are triggered either by the requirements of user or the components of the network itself.

Introducing these dynamic topologies as a key attribute for a WASN, intensifies the necessity to eliminate global knowledge of the network from the perspective of each node. As observed in [5], it is impractical to consider that each node has, or can obtain, awareness of the attributes that characterise the entire network. On the contrary, each node can have access to a set of network characteristics that are limited by the operational range within its neighbourhood. The resulting, relative perspective that each node has on the network may differ from the actual, global network status. Nevertheless, this perspective formulates the basis, on which

Athanasia Panousopoulou is with the Computing Department, Imperial College London, UK. Roy Sterritt is with the Computer Science Research Institute, University of Ulster, UK. Anthony Tzes is with the Faculty of Electrical and Computing Engineering Department, University of Patras, Greece. Corresponding author's email address: a.panousopouloulimperial.ac.uk distributed techniques can be employed for achieving the goals of the entire WASN.

The relative awareness that each node has of the network also benefits the establishment of connectivity across the entire network [3]. Ensuring connectivity is considered essential for the (re)configuration across the WASN. A connected network is a prerequisite for employing more advanced network techniques, such as the construction of Minimum Spanning Trees [6], routing protocols [9], and coverage-oriented schemes for mobile networks [17].

As shown in [4], connectivity conditions are strongly aligned to the spatial attributes of the nodes, while they are achievable when the apposite network model is combined with graph theory tools [15]. Typical decentralised approaches for establishing connectivity are recorded in recent literature either as specialised networking protocols [2], [1] or as feedback- and local observer- based regulation schemes [7], [18]. From an algorithmic perspective, the connectivity problem is modelled either as a Minimum Spanning Tree [8], or a Shortest Path Problem [16], requiring in both cases the exchange of customised messages between the operational nodes.

The issues addressed in this paper are related to the endto-end connectivity over a WASN. End-to-end connectivity refers to the bidirectional connectivity between each pair of nodes in the network. More specifically, relative network and spatial awareness is built for each node, based on its maximum achievable operational range. This awareness is described by a set of relative operational nodes and a relative Delaunay tessellation. As proven herein, the sufficient conditions described in [13], which associate endto-end connectivity to the globally-calculated Delaunay triangulation, can be extended to the case of the relative Delaunay triangulation. These conditions are further used for the synthesis of the Relative Delaunay Connectivity Algorithm. Although this work considers an algorithmic approach, the proposed scheme can be repeatedly employed in an independent and localised manner by each node, in order to calculate the minimum transmission power required to establish end-to-end network connectivity. Theoretical and simulation analysis highlight the efficacy of the resulting transmission power adjustment in end-to-end connectivity terms, whilst introducing no additional network overhead.

The remainder of this paper is organized as follows: in Section II the end-to-end connectivity problem is formulated. In Section III the proposed algorithm is presented, accompanied by the theoretical analysis of its operation. Extensive simulation results are offered in Section IV, while 
the conclusions of this work are drawn in Section V.

\section{Problem Statement}

In this work we consider a WASN, henceforth denoted as $\left(N, \Gamma_{N}\right)$, comprised of a set $\Gamma_{N}=\{1,2, \ldots, N\}$ of $N$ nodes. Each node $i \in \Gamma_{N}$ is solely characterised by: (a) its 2-D coordinates (b) its transmission power $P_{i}^{t x}$, constrained by a predefined upper bound $\rho^{\max }$. Based on this information, the objective is to locally calculate the minimum transmission power that ensures end-to-end network connectivity.

The connectivity status of the network is modelled according to [13]. More specifically, the connectivity of the network is represented as a graph of members of $\Gamma_{N}$, constructed by the bidirectional connectivity links $i \leftrightarrow j$ between the nodal pairs $(i, j)$. The existence of a $i \leftrightarrow j$ link depends on the relative location of nodes $i$ and $j$ and their transmission coverage areas $\mathbf{C}_{i}, \mathbf{C}_{j}$ respectively. The transmission coverage area $\mathbf{C}_{i}$ is represented as a circle, while the corresponding transmission radius is a function of the transmission power $P_{i}^{t x}$, capturing the free space propagation and ground reflection effects [14].

This model reflects the widely accepted notion of binary connectivity [3]. More specifically, the establishment of the bidirectional connectivity link $i \leftrightarrow j$ over the nodal pair $(i, j)$ is quantified by means of the connectivity link binary parameter $\alpha_{i j}$. More specifically, $\alpha_{i j}=1$ if $i \in \mathbf{C}_{j}$ and $j \in \mathbf{C}_{i}$, and $\alpha_{i j}=0$ otherwise.

From the perspective of the entire network, end-to-end connectivity depends on the combination of the aforementioned connectivity links into connectivity paths $P_{i j}=$ $\{i, \ldots, k, \ldots, j\}$ for each nodal pair $(i, j)$. Respective to the connectivity link parameter $\alpha_{i j}$, the existence of such connectivity paths $P_{i j}$ is quantified by means of the connectivity path parameter $b_{i j}$ :

$$
b_{i j} \triangleq 1-\prod_{m=1}^{\left|P_{i j}\right|-1} \alpha_{P_{i j}\{m\} P_{i j}\{m+1\}}=\left\{\begin{array}{l}
0 \text { if }(i, j) \text { is connected } \\
1 \text { otherwise }
\end{array},\right.
$$

where $\left|P_{i j}\right|$ denotes the length of sequence $P_{i j}$ and $P_{i j}\{m\}$ denotes the element of $P_{i j}$ at the $m$-th index.

The binary parameter $b_{i j}$ yields the necessary and sufficient condition for the connectivity of the network:

$$
\sum_{i \in \Gamma_{N}} \sum_{k \in \Gamma_{N}} b_{i k}=0
$$

The condition described by (1) highlights the implicit dependency of the connectivity status of the network on the spatial attributes of its nodes. These spatial attributes can be represented as the Delaunay graph $G_{S}$ [12] of the members of the $\Gamma_{N}$. Graph $G_{S}$ is plane and formulated by Delaunay edges $e_{i j}$ between pairs of nodes $i$ and $j$. The distinct characteristic of a Delaunay edge $e_{i j}$ is that the apposite circumcicle contains no other members of the $\Gamma_{N}$ set [12]. At a nodal level, the vertices of $G_{S}$ that are adjacent to the $i$-th vertex formulate the local set $\Delta_{i}$ of Delaunay neighbours of the $i$-th vertex. As proven in [13], the characteristics of $G_{S}$ formulate the sufficient conditions for the distributed end-toend connectivity of the entire network:

Lemma 1 [13]. If $\forall i \in \Gamma_{N}$ :

$$
\sum_{j \in \Delta_{i}} b_{i j}=0, \text { via } P_{i j}=\left\{\{p, q\} \mid\{p, q\} \equiv e_{p q}, p, q \in\{i\} \cup \Delta_{i}\right\},
$$

then condition (1) is satisfied.

\section{A. The Relative Network and Spatial Awareness}

The set $\Delta_{i}$ of Delaunay neighbours corresponds to a concentrated view on a globally-calculated spatial perspective of the entire network, represented by the graph $G_{S}$. As such, it is impractical to assume that each node has a priori-knowledge of the local set $\Delta_{i}$ of Delaunay neighbours. By contrast, each node is only capable of obtaining a relative view of the spatial configuration of its surrounding co-operational nodes, limited by the maximum transmission power $\rho^{\max }$. Based on this observation, the $i$-th node is capable of deriving the set $\tilde{\Gamma}_{i} \subseteq \Gamma_{N}$ of relative operational nodes, with which it can establish direct connectivity links:

$$
\tilde{\Gamma}_{i} \triangleq\{i\} \bigcup\left\{j \in \Gamma_{N} \mid \alpha_{i j}=1\right\} \text {, at } P_{i}^{t x}, P_{j}^{t x} \leftarrow \rho^{\max } .
$$

Due to the definition of $\alpha_{i j}$, if $i \in \tilde{\Gamma}_{j}$, then $j \in \tilde{\Gamma}_{i}$.

Considering that each node features its 2D-coordinates, the calculation of the set $\tilde{\Gamma}_{i}$ implies that the $i$-th node has access to the related spatial attributes of each $j \in \tilde{\Gamma}_{i}$. Therefore, each node $i$ is capable of deriving the relative Delaunay graph $\tilde{G}_{i}$ and the set $\tilde{\Delta}_{i}$ of relative Delaunay neighbours, similarly to the definition of $G_{S}$ and $\Delta_{i}$, yet solely relying on localised information $\tilde{\Gamma}_{i}$ that it has the potential of acquiring.

From the perspective of the entire network, the relative spatial graph $\tilde{G}_{S}$ is defined as the union of the partially constructed graphs $\tilde{G}_{i}, \forall i \in \Gamma_{N}$. Although $\tilde{G}_{i}$ preserves the properties of a Delaunay tessellation and contrary to the features of graph $G_{S}$, the relative graph $\tilde{G}_{S}$, cannot be considered as either connected or plane. An example of the differences between $G_{S}$ and $\tilde{G}_{S}$ is illustrated in Fig. 1 for a network comprised of $N=34$ operational nodes.
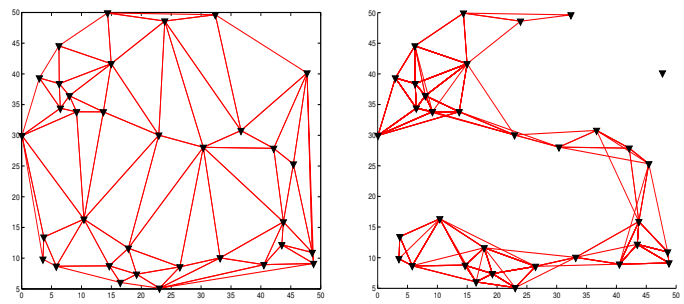

Fig. 1. The spatial graph $G_{S}$ (left) and $\tilde{G}_{S}$ (right) for a network comprised of $N=34$ nodes.

The difference between the graphs $G_{S}$ and $\tilde{G}_{S}$ can be further extended to a nodal perspective. More specifically, a set of properties is derived, characterising both the relationship between the relative Delaunay awareness of two distinct nodes, as well as the relationship between $\tilde{\Delta}_{i}$ and $\Delta_{i}$ for a single node $i$. These properties, illustrated in the example of Fig. 2, are listed as follows:

P1. The set $\tilde{\Delta}_{i}$ of relative Delaunay neighbours is not by definition subset of $\Delta_{i}: \tilde{\Delta}_{i} \nsubseteq \Delta_{i}$.

P2. $\tilde{\Delta}_{i} \equiv \Delta_{i}$ if $\alpha_{i j}=1, \forall j \in \Delta_{i}$, when $P_{i, j}^{t x} \leftarrow \rho^{\max }$.

P3. Relative Delaunay edges are not necessarily symmetric. Equivalently: $j \in \tilde{\Delta}_{i} \nRightarrow i \in \tilde{\Delta}_{j}$.

P4. Symmetric relative Delaunay edges do not imply identical Delaunay triangles, viewed from the extremes of the same symmetric Delaunay edge. For example, if $m \in \tilde{\Delta}_{i}$ 


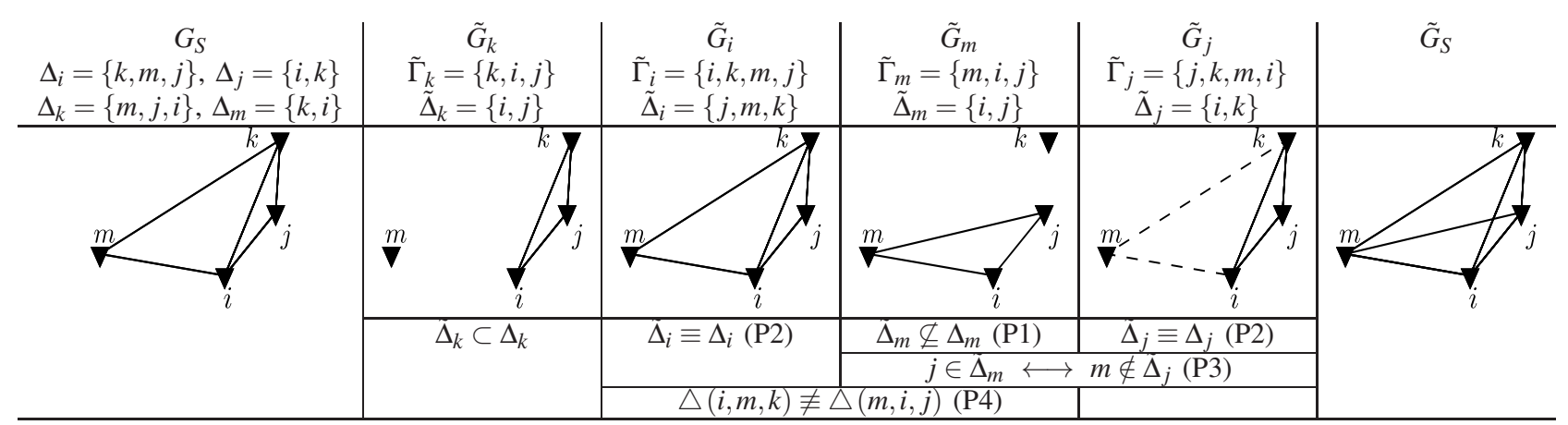

Fig. 2. Example of a spatial graph $G_{S}$ for nodes $i, j, k, m$, the corresponding relative spatial configuration $\tilde{G}_{i}-\tilde{\Delta}_{i}, \tilde{G}_{j}-\tilde{\Delta}_{j}, \tilde{G}_{k}-\tilde{\Delta}_{k}, \tilde{G}_{m}-\tilde{\Delta}_{m}$ for each node, and the resulting spatial graph $\tilde{G}_{S}$. The graph $\tilde{G}_{S}$ for a set of nodes $\Gamma_{N}=\{i, j, k, m\}$ is constructed when direct connectivity links are feasible among all pairs in $\Gamma_{N}$ except for the nodal pair $(k, m)$.

and $i \in \tilde{\Delta}_{m}$ the corresponding Delaunay triangles $\triangle(m, i, j)$, $\triangle(i, m, k)$, viewed from $m$ and $i$ respectively are not necessarily identical. In particular, if $j \notin \tilde{\Gamma}_{i}\left(k \notin \tilde{\Gamma}_{m}\right)$, then $\triangle(m, i, j) \not \equiv \triangle(i, m, k)(j \not \equiv k)$.

The analysis provided so far highlights the fact that, substituting the knowledge $\Delta_{i}$ that each node $i$ has of the globally calculated spatial representation $G_{S}$ with the triple $\tilde{\Gamma}_{i}-\tilde{G}_{i}-\tilde{\Delta}_{i}$ yields different perspectives of the same network, when viewed from different nodes. Nevertheless, this relative spatial and network awareness does not overrule the end-toend connectivity condition for the entire network, described by (1). By contrast, as defined below, each node $i$ undertakes the calculation of the minimum value of its transmission power that satisfies the end-to-end connectivity condition (1), based on its relative network and spatial awareness:

Problem 1. Consider $\left(N, \Gamma_{N}\right)$ WASN. Each node $i \in \Gamma_{N}$ is characterised by its $2 D$ coordinates and its transmission power $P_{i}^{t x}\left(P_{i}^{t x} \leq \rho^{\max }\right)$. Based on the triple $\tilde{\Gamma}_{i}-\tilde{G}_{i}-\tilde{\Delta}_{i}$ of relative network and spatial awareness that the $i$-th node obtains when $P_{i}^{t x} \leftarrow \rho^{\max }$, calculate the minimum value of the transmission power $P_{i}^{t x}, \forall i \in \Gamma_{N}$, that satisfies the endto-end connectivity condition (1).

\section{END-TO-END CONNECTIVITY BASED ON RELATIVE DELAUnAY AWARENESS}

The establishment of end-to-end connectivity across the entire network from a distributed, nodal perspective can be achieved by utilising the relative Delaunay $\tilde{\Delta}_{i}$ awareness of the $i$-th node. The constraint introduced is related to the characteristics of the spatial graph $G_{S}$. In particular, the end-to-end connectivity across the entire network is achievable if $\tilde{G}_{S}$ is connected. This condition yields an essential requirement for extending the $\Delta_{i}$-related sufficient end-to-end connectivity condition ( $\left.\tilde{\Delta}_{i}\right)$ to the case of relative Delaunay awareness, described by $\tilde{\Delta}_{i}$.

Lemma 2. Consider a $\left(N, \Gamma_{N}\right)$ WASN, described by the relative spatial graph $\tilde{G}_{S}$. If

$$
\sum_{j \in \tilde{\Delta}_{i}} b_{i j}=0, \text { via } P_{i j}=\left\{\{p, q\} \mid\{p, q\} \equiv e_{p q}, p, q \in\{i\} \bigcup \tilde{\Delta}_{i}\right\}
$$

over a connected spatial graph $\tilde{G}_{S}$, then condition (1) holds. Proof. The proof of Lemma 2 is similar to the proof of Lemma 1 [13], and it is, thereby, omitted.

It should be noted that the sufficient conditions described by (2) are not necessary for the end-to-end connectivity over
$\Gamma_{N}$; if condition (1) holds, the connectivity path among two relative Delaunay neighbours is not necessarily constructed by relative Delaunay edges. Nevertheless, as shown below, Lemma 2 provides the roadmap for solving Problem 1.

\section{A. The Relative Delaunay Connectivity Algorithm}

The sufficient connectivity conditions described by Lemma 2 imply that knowledge of the relative Delaunay neighbourhood $\widetilde{\Delta}_{i}$, along with its accompanying tessellation, is adequate for calculating the minimum transmission power required to establish end-to-end network connectivity. More specifically, the Distributed Delaunay Connectivity Algorithm (DDelCA), presented and evaluated in [13] for the case of the local Delaunay sets $\Delta_{i}$, can be employed when the $i$-th node has a relative Delaunay awareness, constrained by its maximum achievable range

The resulting Relative Delaunay Connectivity Algorithm (ReDelCA) is summarised as a two-step process. During Step 1 , the value $\rho_{i j}$ of $P_{i}^{t x}$, required for realising the shortest path $\pi_{i j}$ towards the $j$-th relative Delaunay neighbour, is calculated. This calculation is based on $\tilde{\Delta}_{i}\left(p, q, t \in\{i\} \cup \tilde{\Delta}_{i}\right)$ and the apposite tessellation $\left(\{p, q\} \equiv e_{p q},\{q, t\} \equiv e_{q t}\right)$. The path $\pi_{i j}=\{i, \ldots, p, q, t \ldots, j\}$ is constructed by the $t$-th relative Delaunay neighbours that minimise the label $\left.c_{l}(t)\right|_{q}$ :

$$
\left.c_{l}(t)\right|_{q} \triangleq c(q)+P_{q t}^{t x} .
$$

In (3) the vertex $q$ is defined as the origin node of vertex $t$, while $P_{q t}^{t x}$ is the value of the transmission power required on behalf of node $q$ to establish a link with node $t$. Considering the case of symmetric links, this implies $P_{q t}^{t x} \equiv P_{t q}^{t x}$. In addition, if $P_{t q}^{t x}>\rho^{\max }$, the link $q \leftrightarrow t$ is not feasible. Finally, the term $c(q)$ denotes the length function at the $q$-th vertex:

$$
c(q) \triangleq c(p)+\max \left\{P_{q p}^{t x}, P_{q t}^{t x}\right\} .
$$

The value $c(q)$ of the length function is associated with: (a) the transmission power $P_{q p}^{t x}, P_{q t}^{t x}$ that node $q$ should use to establish with nodes $p$ and $t$, respectively, (b) the value of the length function at the $c(p)$ at the $p$-th vertex. Finally, if $q \equiv i$, then $c(q)=P_{i t}^{t x}$.

During Step 2, the value $\rho_{i}^{t x}\left(\leq \rho^{\max }\right)$ is set to the value that is capable of realising the shortest path $\pi_{i j}$, that the $i$-th 
node calculates at Step 1 for each $j \in \tilde{\Delta}_{i}[13]$ :

$$
\rho_{i}^{t x}=\min \left\{\rho^{\max } \max _{j \in \tilde{\Delta}_{i}}\left\{\rho_{i j}^{t x}\right\}\right\} .
$$

Both the calculation of $\pi_{i j}$, which minimises the transmission power required to establish the $i \leftrightarrow j$ link, as well as the selection of the $\rho_{i}^{t x}$, according to (5), are executed independently at each node. The design approach of ReDelCA reveals also its repeatability within a varying network topology, as it is based on completely localised information, introducing neither additional complexity nor network overhead. Moreover, as in the case of DDelCA, RDelCA converges in quadratic time $\left(O\left(N^{2}\right)\right)$. Finally, as discussed below, setting $P_{i}^{t x} \leftarrow \rho_{i}^{t x}, \forall i \in \Gamma_{N}$ satisfies condition (1), and thereby, solves Problem 1 under the assumption of a connected graph $\tilde{G}_{S}$.

\section{B. Analysis of the Relative Delaunay Connectivity Algorithm}

The backbone of the ReDelCA is the calculation of the shortest path $\pi_{i j}, \forall j \in \tilde{\Delta}_{i}$, that minimises the transmission power required by the $i$-th node to establish a link with its $j$-th relative Delunay neighbour, via the relative Delaunay edges of the $i$-th node. As each node has a different perspective on the same network, the independent and localised execution of the ReDelCA at different nodes $i, j$ cannot guarantee that the corresponding paths $\pi_{i j}$ and $\pi_{j i}$ will be symmetric. Nevertheless, this property is proven for the case of a shortest path that is comprised of a single and symmetric relative Delaunay edge.

Lemma 3. If $\pi_{i j}=\{i, j\}$ and $i \in \tilde{\Delta}_{j}$, then $\pi_{j i}=\{j, i\}$.

Proof. Since $\pi_{i j}=\{i, j\}, j \in \tilde{\Gamma}_{i}$ and, thus, $P_{i j}^{t x} \leq \rho^{\max }$. In addition, for $k \in \tilde{\Delta}_{i}$, which determines the triangle $\triangle(i, j, k)$ :

$$
\left.c_{l}(j)\right)\left.\right|_{i}<\left.c_{l}(j)\right|_{k} \Rightarrow 2 P_{i j}^{t x}<\left.c_{l}(j)\right|_{k} .
$$

Based on (3)-(4), (6) implies that $\forall o(k) \in\{i\} \bigcup \tilde{\Delta}_{i}$ :

$$
\begin{aligned}
& 2 P_{i j}^{t x}<\left.c_{l}(k)\right|_{o(k)}+\max \left\{P_{o(k) k}^{t x}, P_{k j}^{t x}\right\}+P_{k j}^{t x} \Rightarrow \\
& 2 P_{i j}^{t x}<P_{i k}^{t x}+\max \left\{P_{i k}^{t x}, P_{k j}^{t x}\right\}+P_{k j}^{t x}, \text { when } o(k)=i .
\end{aligned}
$$

Assume that $\pi_{j i} \neq\{j, i\}$. In this case $\pi_{j i}$ is formulated as $\pi_{j i}=\left\{j, \ldots, k^{\prime}, i\right\}$, where $k^{\prime} \in \tilde{\Delta}_{j}$ and $\left(i, j, k^{\prime}\right)$ determines the Delaunay triangle $\triangle\left(j, i, k^{\prime}\right)$ from the perspective of the $j$-th node. Since $\pi_{j i} \neq\{j, i\}$ :

$$
\left.c_{l}(i)\right|_{k^{\prime}}<2 P_{i j}^{t x} \Rightarrow 2 P_{i j}^{t x}>P_{i k^{\prime}}^{t x}+\max \left\{P_{i k^{\prime}}^{t x}, P_{k^{\prime} j}^{t x}\right\}+P_{k^{\prime} j}^{t x} .
$$

The following cases are examined:

A. $k^{\prime} \in \tilde{\Delta}_{i} \Rightarrow k^{\prime} \equiv k$. In this case condition (7) contradicts to condition (8) and, thus, the assumption that $\pi_{j i} \neq\{j, i\}$ is negated.

B. $k^{\prime} \notin \tilde{\Delta}_{i} \Rightarrow k \not \equiv k^{\prime}$. Due to P4, $k^{\prime} \notin \tilde{\Gamma}_{i}$. Consequently, the transmission power $P_{i k^{\prime}}^{t x}$ is constrained by the upper threshold $\rho^{\max }$. This observation leads to:

$$
\begin{aligned}
P_{i k^{\prime}}^{t x} & >\rho^{\max } \Rightarrow P_{i k^{\prime}}^{t x}+\max \left\{P_{i k^{\prime}}^{t x}, P_{k^{\prime} j}^{t x}\right\}+P_{k^{\prime} j}^{t x}>3 \rho^{\max } \Rightarrow \\
2 P_{i j}^{t x} & >P_{i k^{\prime}}^{t x}+\max \left\{P_{i k^{\prime}}^{t x}, P_{k^{\prime} j}^{t x}\right\}+P_{k^{\prime} j}^{t x}>3 \rho^{\max }>2 \rho^{\max },
\end{aligned}
$$

due to (8). Inequality (9) violates the constraint $P_{i j}^{t x}<\rho^{\max }$. Therefore, the assumption that $\pi_{j i} \neq\{j, i\}$ is negated. Consequently, if $\pi_{i j}=\{i, j\}$ and $i \in \Delta_{j}$, then $\pi_{j i}=\{j, i\}$.
Based on Lemma 3, Corollaries 1, 2 and 3 are derived: Corollary 1. If $\pi_{i j}=\{i, k, \ldots, j\}$ and $i \in \tilde{\Delta}_{k}$, then $\pi_{i k}=\{i, k\}$. Corollary 2.If $\pi_{i j}=\{i, j\}$ and $i \in \tilde{\Delta}_{j}$, then $\alpha_{i j}=1$ for $P_{i}^{t x} \leftarrow$ $\rho_{i}^{t x}, P_{j}^{t x} \leftarrow \rho_{j}^{t x}$.

Corollary 3. If $\pi_{i k}=\{i, j, k\}$, for $i \in \tilde{\Delta}_{k} \cap \tilde{\Delta}_{j}$ and $j \in \tilde{\Delta}_{k}$, $k \in \tilde{\Delta}_{j}$, then $\pi_{j k} \neq\{j, i, k\}$.

Lemma 3 and the resulting Corollaries are utilised to prove that the transmission power $\rho_{i}^{t x}$, which results from employing ReDelCA at each node $i \in \Gamma_{N}$, yields end-to-end network connectivity over a connected $\tilde{G}_{S}$ :

Theorem 1. Consider a $\left(N, \Gamma_{N}\right) W A S N$, described by a connected spatial graph $\tilde{G}_{S}$. If $P_{i}^{t x}=\rho_{i}^{t x}, \forall i \in \Gamma_{N}$, then condition (1) is satisfied.

Proof. Due to Lemma 2, it is sufficient to prove that when $P_{i}^{t x}=\rho_{i}^{t x}$ for each $i \in \Gamma_{N}$ over a connected spatial graph $\tilde{G}_{S}$ then the connectivity condition described by (2) is satisfied within the relative Delaunay neighbourhood.

Without loss of generality, we consider a network comprised of four nodes $\Gamma_{N}=\{i, m, k, j\}$ and characterised by $\tilde{G}_{S}$ shown in Fig. 2. The relative spatial awareness per node is resumed bellow:

$$
\begin{array}{l|l}
i: \tilde{\Gamma}_{i}=\{i, m, k, j\}, \tilde{\Delta}_{i}=\{m, k, j\} & m: \tilde{\Gamma}_{m}=\{m, i, j\}, \tilde{\Delta}_{m}=\{i, j\} \\
\hline k: \tilde{\Gamma}_{k}=\{k, i, j\}, \tilde{\Delta}_{k}=\{i, j\} & j: \tilde{\Gamma}_{j}=\{j, i, m, k\}, \tilde{\Delta}_{j}=\{i, k\}
\end{array}
$$

The following cases are examined:

A. Symmetric Delaunay Edges: $i \in \tilde{\Delta}_{k}$ and $k \in \tilde{\Delta}_{i}$.

Let us assume that $\alpha_{i k}=0$, when the $i$-th and $k$-th transmission power is set to $\rho_{i}^{t x}, \rho_{k}^{t x}$ respectively. This implies that $\pi_{i k} \neq\{i, k\}$, and, thus, $\pi_{i k}$ is equal to either $\{i, j, k\}$, or $\{i, m, k\}$. Considering the path $\pi_{i k}=\{i, m, k\}$ leads to:

$$
\begin{aligned}
2 P_{i k}^{t x} & >\left.c_{l}(k)\right|_{m}, \text { and } \\
\left.c_{l}(k)\right|_{m} & =P_{i m}^{t x}+\max \left\{P_{i m}^{t x}, P_{m k}^{t x}\right\}+P_{m k}^{t x} \geq 2 P_{m k}^{t x} \Rightarrow \\
\left.c_{l}(k)\right|_{m} & >2 \rho^{\max },
\end{aligned}
$$

as $m \notin \tilde{\Gamma}_{k}$, or equivalently $P_{m k}^{t x}>\rho^{\max }$. Equations (10)(11) imply that $2 P_{i k}^{t x}>2 \rho^{\max }$, which violates the initial assumption that $k \in \Gamma_{i}$. Therefore, $\pi_{i k}=\{i, j, k\}$, which leads to:

$$
\pi_{i k}=\{i, j, k\} \Rightarrow \pi_{i j}=\{i, j\} \Rightarrow \alpha_{i j}=1,
$$

due to Corollary 1 and Corollary 2 respectively. In addition:

$$
\pi_{i k}=\{i, j, k\} \Rightarrow \pi_{j k}=\{j, k\} \Rightarrow \alpha_{j k}=1,
$$

due to Corollary 3 and Corollary 2 respectively. As a result, for $P_{i}^{t x} \leftarrow \rho_{i}, P_{j}^{t x} \leftarrow \rho_{j}$ and $P_{k}^{t x} \leftarrow \rho_{k}$, the connectivity path $P_{i k}=\{i, j, k\}$ is characterised by $b_{i j}=1-\alpha_{i j} \alpha_{j k}=0$. Thus, nodes $i$ and $k$ are connected.

B. Non-symmetric Delaunay Edges: $j \in \tilde{\Delta}_{m}$ and $m \notin \tilde{\Delta}_{j}$.

As previously shown $\alpha_{i j}=1$, thus, a valid connectivity path $P_{m j}=\{m, i, j\}$ ensuring that $b_{m j}=0$ exists if $\alpha_{i m}=1$. Considering that $m \in \Delta_{i}$, this implies that it is sufficient to show that $\pi_{i m}=\{i, m\}$.

Let us assume the opposite, i.e. that $\pi_{i m} \neq\{i, m\}$. Consequently, path $\pi_{i m}$ is either equal to $\{i, j, k, m\}$ or to $\{i, k, m\}$. This observation leads subsequently to:

$$
\begin{aligned}
& 2 P_{i n}^{t x}>\left.c_{l}(m)\right|_{k} \Rightarrow \\
& 2 P_{i m}^{t x}>c(o(k))+\max \left\{P_{o(k) k}^{t x}, P_{k m}^{t x}\right\}+P_{k m}^{t x}, \text { where } o(k)=\{j, i\} .
\end{aligned}
$$

Therefore $2 P_{i m}^{t x}>2 P_{k m}^{t x}$. This inequality is invalid, since $P_{i m}^{t x} \leq \rho^{\max }\left(m \in \tilde{\Gamma}_{i}\right)$ and $P_{m k}^{t x}>\rho^{\max }\left(k \notin \tilde{\Gamma}_{m}\right)$. As such, the 
initial assumption that $\pi_{i m} \neq\{i, m\}$ is negated, and thereby $\alpha_{i m}=1$. Consequently, $b_{m j}=0$, via the connectivity path $P_{m j}=\{m, i, j\}$, when the transmission power $P_{i}^{t x}, P_{j}^{t x}$ and $P_{m}^{t x}$ equals to $\rho_{i}^{t x}, \rho_{j}^{t x}$ and $\rho_{m}^{t x}$ respectively. $\square$

It should be noted that the localised policy of ReDelCA yields optimal conditions constrained to the relative awareness that each node has on the network. With respect to global perspective of the network, ReDelCA provides a suboptimal approach on the solution for Problem 1. Nevertheless, as shown in Section IV, the gap among the proposed sub-optimal conditions and some optimal conditions is not severe.

\section{Simulation Studies}

The proposed scheme calculates the minimum transmission power required for end-to-end connectivity. Although the minimum transmission power does not necessarily yield the optimal configuration for a power-conservative and varying network, its awareness is essential as it provides the connectivity potentiality of the network. Based on this observation, the evaluation of the network behaviour that results from applying the solution $\rho_{i}^{t x}$ of ReDelCA at the transmission power $P_{i}^{t x}, \forall i \in \Gamma_{N}$ relies on an extensive simulation procedure. This procedure employs 10 different cases of normal distribution on the nodes deployment per network size $N=\{4,9,14, \ldots, 299\}$, within a fixed bounded $2 \mathrm{D}$ region. The network topology is not considered static, however during the calculation of $\rho_{i}^{t x}$ the relative network and spatial awareness per node are assumed to be constant.

The performance of a network resulting from the transmission power configuration based on ReDelCA is evaluated against the performance of the same network that results from: (a) a transmission power configuration with $P_{i}^{t x}=$ $\rho^{\max }$, (b) the transmission power configuration based on DDelCA [13], (c) the transmission power configuration based on the Localised Minimum Spanning Tree (LMST) [8].

With respect to the connectivity status, the evaluation metrics for the performance of the network are (a) the number of disjoint parts of the network (clusters) and (b) the number of direct connectivity links. The behaviour of the network is additionally evaluated against the mean value of the lifetime $\tau_{i}$. More specifically, the calculation of the lifetime $\tau_{i}$ is based on the estimation model presented in [10], considering unslotted CSMA-CA as the medium access mechanism, and fixed transmission $f_{T X}$ and reception $f_{R X}$ rate per duty cycle, while each node utilises the local broadcasting model.

\section{A. Simulation Results}

An example of the connectivity status of a network comprised of $N=54$ nodes for the aforementioned cases of transmission power configuration is shown in Fig. 3. All schemes examined offer end-to-end connectivity. On the contrary to the network resulting from the maximum transmission power configuration (Fig. 3(a)), the ReDelCAbased network configuration (Fig. 3(d)) accomplishes end-toend connectivity without introducing extraordinary network links. The different perspective on the spatial information results into slightly different network connectivity status when DDelCa (Fig. 3(c)) and ReDelCA are employed. Nevertheless, in both cases the redundant edges are a result of the localised nature of these algorithms, contrasting the optimal configuration described by the LMST-based configuration (Fig. 3(b)). This paradigm highlights that despite the suboptimality offered by ReDelCA, the gap between the apposite solution and the solution provided by LMST is not severe.

1) End-to-End Connectivity: The mean value of isolated clusters and the mean value of direct connectivity links are are presented in the left and middle diagram of Fig. 4 respectively. As shown in the left diagram of Fig. 4, the connectivity status achieved when the transmission power configuration is based on ReDelCA is identical to the connectivity status achieved when the three remaining benchmarking algorithms are employed for configuring the transmission power of the network. This implies that relative Delaunay awareness, with no additional network overhead, is adequate for establishing end-to-end connectivity across the entire network, given that connectivity is achievable when nodes operate at their maximum allowable transmission power.

The benefits of the ReDelCA are additionally highlighted in the middle diagram of Fig. 4, whereby the mean value of direct connectivity links is presented. The end-to-end connectivity status that relies on the LMST-based transmission power configuration results into the most minimalistic network graph, with the expense of introducing additional network overhead in the operation of the network. The connectivity status of the network resulting from ReDelCA converges to the connectivity status of the network resulting from DDelCA, as the network size increases. This observation implies that as the network becomes more dense, the relative Delaunay awareness converges to the globallyderived Delaunay awareness $\left(\tilde{\Delta}_{i} \equiv \Delta_{i}\right)$.

2) Lifetime: The mean value of lifetime $\tau_{i}$ per node is presented at the right diagram of Fig. 4, highlighting the benefits that transmission power configuration based on ReDelCA has on the mean value of the nodal lifetime. When $N \leq 150$, the transmission power configuration resulting from RelDelCA yields better lifetime performance than the nodal lifetime resulting from the DDelCA-based transmission power configuration. Nevertheless, as the network becomes more dense $(N>150)$, the mean value of $\tau_{i}$ resulting from the employment of ReDelCA converges to the mean value of $\tau_{i}$ when either LMST or DDelCA are utilised for the transmission power configuration.

\section{Conclusions}

In this work, end-to-end connectivity issues have been addressed for the case of a WASN with dynamic attributes, modelled by the relative spatial and network awareness per node. The Relative Delaunay Connectivity Algorithm, presented herein, is employed by each node to calculate the transmission power required to establish end-to-end connectivity. Under the assumption that connectivity of the network is achievable when all nodes operate at their maximum transmission power, this localised transmission range 
(a)

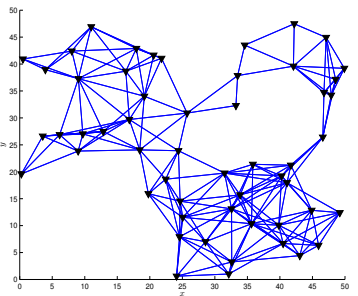

(b)

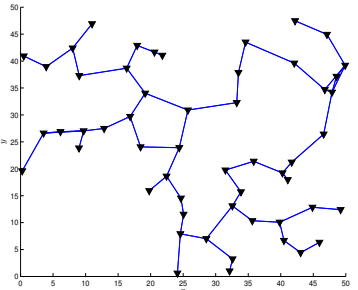

(c)

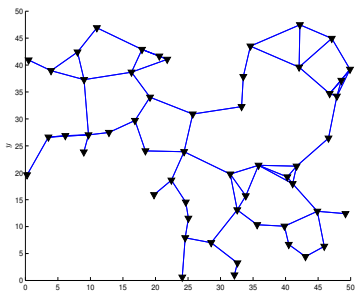

(d)

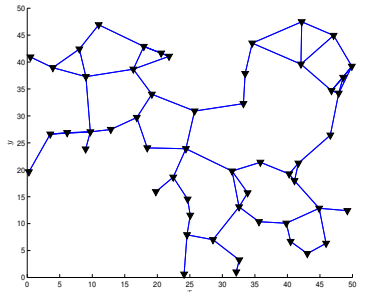

Fig. 3. The connectivity status of a network comprised of $N=54$ nodes, resulting from the transmission power configuration based on (a) static / maximum transmission power, (b) the LMST, (c) the DDelCA and (d) ReDelCA.
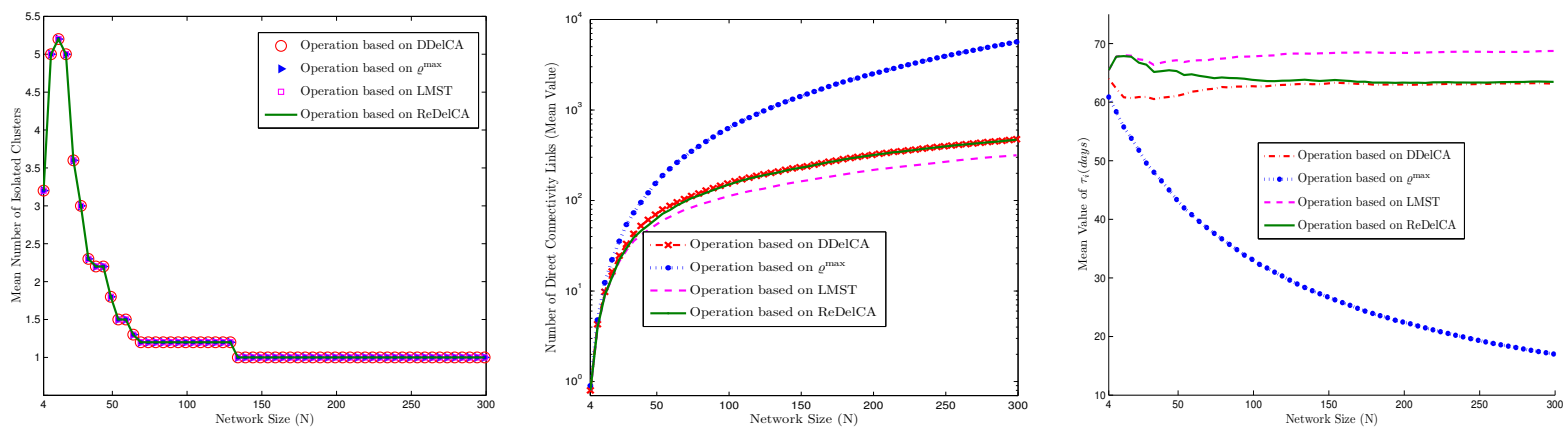

Fig. 4. Simulation results: The mean value of isolated clusters (left), the mean value of direct connectivity links (middle), the mean lifetime per node (right) for $f_{R X}=f_{T X}=1000 \mathrm{bps}$, versus the network size $N$.

adjustment satisfies the end-to-end connectivity conditions. The simulation results empower the theoretical outcome, highlighting the advantages that the proposed scheme has in terms of connectivity and lifetime.

The consideration of temporal characteristics that eliminate the symmetrical character from the connectivity links yield the roadmap for further research, therefore introducing a more realistic approach, in terms of autonomic network reconfiguration.

\section{ACKNOWLEDGEMENTS}

This work was supported by the Computer Science Research Institute, University of Ulster, UK.

\section{REFERENCES}

[1] J. Cartigny, F. Ingelrest, D. Simplot-Ryl, and I. Stojmenovic, "Localized lmst and rng based minimum-energy broadcast protocols in ad hoc networks," Ad Hoc Networks, vol. 3, no. 1, pp. 1 - 16, 2005.

[2] L. H. A. Correia, D. F. Macedo, A. L. dos Santos, A. A. F. Loureiro, and J. M. S. Nogueira, "Transmission power control techniques for wireless sensor networks," Computer Networks, vol. 51, pp. 47654779, 2007.

[3] A. Ghosh and S. K. Das, "Coverage and Connectivity Issues in Wireless Sensor Networks: A survey "Pervasive and Mobile Computing, vol. 4, pp. 303-334, 2008.

[4] P. Gupta and P. R. Kumar, "Critical power for asymptotic connectivity," in Decision and Control, 1998. Proceedings of the 37th IEEE Conference on, vol. 1, 1998, pp. 1106-1110.

[5] M. Haenggi, J. G. Andrews, F. Baccelli, O. Dousse, and M. Franceschetti, "Stochastic geometry and random graphs for the analysis and design of wireless networks," Selected Areas in Communications, IEEE Journal on, vol. 27, no. 7, pp. 1029-1046, 2009.

[6] M. Khan, G. Pandurangan, and V. S. Anil Kumar, "Distributed Algorithms for Constructing Approximate Minimum Spanning Trees in Wireless Sensor Networks," IEEE Transactions on Parallel and Distributed Systems, vol. 20, no. 1, pp. 124-139, 2009.
[7] P. Kumar, "New technological vistas for systems and control: the example of wireless networks," Control Systems, IEEE, vol. 21, no. 1, pp. $24-37,2001$

[8] N. Li, J. C. Hou, and L. Sha, "Design and analysis of an mstbased topology control algorithm," Wireless Communications, IEEE Transactions on, vol. 4, no. 3, pp. 1195-1206, 2005.

[9] X.-Y. Li, G. Calinescu, P. Wan, and Y. Wang, "Localized Delaunay Triangulation with Application in Ad-Hoc Wireless Networks," IEEE Transactions on Parallel and Distributed Systems, vol. 14, pp. 1-13, 2003.

[10] V. P. Mhatre, C. Rosenberg, D. Kofman, R. Mazumdar, and N. Shroff, "A Minimum Cost Heterogeneous Sensor Network with a Lifetime Constraint," IEEE Transcations on Mobile Computing, vol. 4, pp. 4 15, 2005.

[11] R. Min, M. Bhardwaj, S.-H. Cho, N. Ickes, E. Shih, A. Sinha, A. Wang, and A. Chandrakasan, "Energy-centric enabling technologies for wireless sensor networks," IEEE Wireless Communications, vol. 9, no. 4, pp. 28-39, 2002.

[12] A. Okabe, B. Boots, K. Sugihara, and S. N. Chiu, Spatial tessellations: Concepts and applications of Voronoi diagrams, 2nd ed., ser. Probability and Statistics. NYC: Wiley, 2000, 671 pages.

[13] A. Panousopoulou and A. Tzes, "Rf-power overlapping control for connectivity awareness in wireless ad-hoc and sensor networks," in 2nd IFAC Workshop on Distributed Estimation and Control in Networked Systems (NecSys 2010), 2010, pp. 275-280.

[14] T. S. Rappaport, Wireless Communications: Principles and Practice. Prentice Hall, 1996.

[15] M. Sánchez, P. Manzoni, and Z. J. Haas, "Determination of Critical Transmission Range in Ad-hoc Networks," in Proceedings of Multiaccess Mobility and Teletraffic for Wireless Communications Workshop, 1999.

[16] Y. Shen, Y. Cai, and X. Xu, "A shortest-path-based topology control algorithm in wireless multihop networks," SIGCOMM Comput. Commun. Rev., vol. 37, pp. 29-38, 2007.

[17] J. Stergiopoulos and A. Tzes, "Decentralized communication range adjustment issues in multi-agent mobile networks," in American Control Conference (ACC), 2010, 2010, pp. 1629-1634.

[18] M. M. Zavlanos and G. J. Pappas, "Distributed Connectivity Control of Mobile Networks," IEEE Transcactions on Robotics, vol. 24, no. 6 , pp. 1416-1428, 2008. 